\title{
Apparent phase transitions in finite one-dimensional sine-Gordon lattices
}

\author{
Saúl Ares, ${ }^{*}$ José A. Cuesta ${ }^{\dagger}$ and Angel Sánchez \\ Grupo Interdisciplinar de Sistemas Complejos (GISC) and Departamento de Matemáticas, Universidad Carlos III de Madrid, \\ Avenida de la Universidad 30, 28911 Leganés, Madrid, Spain ${ }^{\S}$ \\ Raúl Toral ${ }^{\|}$ \\ Institut Mediterrani d'Estudis Avançats (IMEDEA), CSIC-Universitat de les Illes Balears, \\ Campus UIB, 07071 Palma de Mallorca, Spain ${ }^{\text {Il }}$
}

(Received 15 November 2002; published 17 April 2003)

\begin{abstract}
We study the one-dimensional sine-Gordon model as a prototype of roughening phenomena. In spite of the fact that it has been recently proven that this model cannot have any phase transition [J. A. Cuesta and A. Sánchez, J. Phys. A 35, 2373 (2002)], Langevin as well as Monte Carlo simulations strongly suggest the existence of a finite temperature separating a flat from a rough phase. We explain this result by means of the transfer operator formalism and show as a consequence that sine-Gordon lattices of any practically achievable size will exhibit this apparent phase transition at unexpectedly large temperatures.
\end{abstract}

DOI: 10.1103/PhysRevE.67.046108 PACS number(s): 05.70.Fh, 05.10.-a, 68.35.Ct, 64.60.Cn

\section{INTRODUCTION}

More than 50 years ago, van Hove [1] proved that true thermodynamic phase transitions, defined as singularities of the free energy, could not occur in a class of one-dimensional (1D) systems, a result later extended to lattice systems in the same class by Ruelle [2]. In spite of the fact that the conditions for van Hove's theorem to apply were clearly stated [1] (see also Ref. [3]), there is nowadays a very general belief that 1D systems cannot exhibit phase transitions unless they have long range interactions. This misinterpretation of van Hove's mathematical results has been reinforced by the abuse of Landau's [4] argument about the entropic contribution of domain walls to the free energy. This argument, being physically very intuitive and useful, is not a rigorous result (assumptions such as a dilute concentration of domain walls are made along the way) and, furthermore, it does not apply to every 1D system. In fact, there are many examples of 1D systems with true thermodynamic phase transitions [5-9] which, unfortunately, have remained largely unnoticed.

In the more specific context of models of growth processes $[10,11]$, the unsustained belief discussed above is often translated by saying that $1 \mathrm{D}$ interfaces are always rough. This is actually not the case, as shown in the early eighties with several examples [7,8]. Only recently, two of us [12] have proven a theorem showing rigorously that a wide family of $1 \mathrm{D}$ models, including the sine-Gordon model as a particular example, cannot have phase transitions (some nonrigorous, phenomenological arguments in the same direction had been proposed earlier [13]). However, as we will see below, this theorem turns out to be in conflict with some

\footnotetext{
*Electronic address: saul@math.uc3m.es

${ }^{\dagger}$ Electronic address: cuesta@math.uc3m.es

\#Electronic address: anxo@math.uc3m.es

${ }^{\S}$ URL: http://gisc.uc3m.es

"Electronic address: raul@imedea.uib.es

IIURL: http://www.imedea.uib.es
}

numerical simulation results that seem to provide strong evidence supporting the existence of a roughening phase transition in the 1D sine-Gordon model. In view of the fact that simulations are very often the only way of studying a large class of models, it is most important to understand this contradiction in order to distinguish between true and apparent phase transitions.

To the above end, in this paper we focus on the 1D sineGordon model as a canonical example, widely applicable and representative of the phenomenology of many model systems [14] (see also Ref. [15] for a review). Thus, in Sec. II we give results of simulations that suggest the existence of a phase transition at a (not necessarily small) nonzero temperature. By means of a transfer operator approach and using the probabilistic meaning of the corresponding eigenfunctions, in Sec. III we analyze the origin of this behavior; from this analysis, we are able to conclude that such apparent phase transitions will occur not only for lattice sizes achievable within the present computational capabilities, but also for very much larger lattices. Finally, in Sec. IV we discuss the consequences of this result, which we believe are relevant for computational studies where no analytical support exists. Furthermore, additional important implications of our research for experimental studies of small systems far from the thermodynamic limit are also considered.

\section{NUMERICAL SIMULATIONS}

The 1D sine-Gordon model is defined by the following Hamiltonian:

$$
\mathcal{H}=\sum_{i=1}^{N}\left\{\frac{J}{2}\left(h_{i-1}-h_{i}\right)^{2}+V_{0}\left[1-\cos \left(h_{i}\right)\right]\right\},
$$

where $N$ is the number of lattice nodes (or the system size), $J$ is the coupling constant, and $-\infty<h_{i}<\infty$ is a real variable on site $i$. We assume periodic boundary conditions $h_{0} \equiv h_{N}$. For visualization of our results, we interpret $h_{i}$ as the height 
of a surface above site $i$ of the lattice; then, the two terms of the Hamiltonian correspond, respectively, to surface tension and to a local potential (of strength $V_{0}$ ) favoring multiple values of $2 \pi$ for the height, representing that growth takes place preferentially by addition of discrete units (layers). For surface growth on two-dimensional (2D) substrate lattices, this interpretation has proven itself rather fruitful in the past (see Refs. [16-19] and references therein). However, we want to stress that the results we present in this paper are independent of any specific interpretation one makes of $h_{i}$. In fact, previous studies of the 1D sine-Gordon lattice $[14,20,21]$ were more interested in understanding the role of solitons in statistical mechanics models [15] than in any particular application.

In our study, we concentrate on two magnitudes in order to characterize the model behavior: the surface width or roughness,

$$
w^{2}=\left\langle\frac{1}{N} \sum_{i=1}^{N}\left[h_{i}-\bar{h}\right]^{2}\right\rangle,
$$

where

$$
\bar{h} \equiv \frac{1}{N} \sum_{i=1}^{N} h_{i}
$$

is the mean height, and the height-difference correlation function,

$$
C(r)=\left\langle\frac{1}{N} \sum_{j=1}^{N}\left[h_{r+j}-h_{j}\right]^{2}\right\rangle .
$$

Averages $\langle\ldots\rangle$ are to be understood with respect to a statistical weight given by the Gibbs factor, $e^{-\mathcal{H} / T}$, at equilibrium at a temperature $T$.

The above defined are crucial quantities in the $2 \mathrm{D}$ version of the model. This exhibits a Kosterlitz-Thouless-type phase transition from a low temperature flat phase to a high temperature rough phase $[17,19,22-24]$. In the flat phase, small systems have a size dependent width, whereas the width of large systems is independent of the size. The crossover system size separating both regimes is closely related to the correlation length, which is finite in the low temperature phase, and can be defined as the distance beyond which the height-difference correlation function saturates. On the contrary, in the rough phase the correlation length is infinite and, correspondingly, the roughness increases (logarithmically in the 2D case) with the system size for all sizes, i.e., it is also infinite in the thermodynamic limit. In $1 \mathrm{D}$, the theorem proved in Ref. [12] prohibits any phase transition, and at all nonzero temperatures the system is in the rough phase, the roughness increasing linearly with the system size.

In the lack of detailed analytical results, the statistical averages can be computed approximately by means of numerical simulations. This kind of analysis has become a routine tool for the study of the equilibrium properties of many models and a problem of interest is to extract from the numerical studies, necessarily performed in finite size lattices, the asymptotic behavior in the thermodynamic limit. We will show that a naïve extrapolation of the finite size results for the 1D sine-Gordon model can lead to erroneous results concerning the existence of a phase transition at a finite value of the temperature.

For our numerical study, and in order to assess the validity of our results, we have used two completely different procedures: Langevin dynamics and parallel tempering Monte Carlo. The Langevin dynamics procedure has been widely used in this context with very good results [17-19], and it consists of the numerical integration of the Langevin equation following from the Hamiltonian $\mathcal{H}$ :

$$
\frac{d h_{i}(t)}{d t}=-\frac{\partial \mathcal{H}}{\partial h_{i}(t)}+\eta_{i}(t),
$$

where $\eta_{i}$ are Gaussian white noises obeying the fluctuationdissipation theorem at temperature $T$, i.e.,

$$
\left\langle\eta_{j}\left(t^{\prime}\right) \eta_{i}(t)\right\rangle=2 T \delta_{i j} \delta\left(t-t^{\prime}\right) .
$$

A major problem with the Langevin dynamics is the presence of systematic errors, in addition to the unavoidable statistical errors, due to the finiteness of the time step used in the numerical integration (in our studies, we have used a stochastic Heun method [25]).

The second procedure, parallel tempering, is the one we have mostly relied on. The reason is that it is a very efficient algorithm to prevent the system from being trapped in local minimum energy configurations. Parallel tempering requires any Monte Carlo method that generates representative configurations at a given temperature. In this case, we have implemented a heat bath algorithm [26], in which new values $h_{i}^{\prime}$ for the height at site $i$ are proposed according to the rule

$$
h_{i}^{\prime}=\frac{h_{i-1}+h_{i+1}}{2}+\xi \sqrt{\frac{T}{2 J}},
$$

$\xi$ being a Gaussian random variable of zero mean and unit variance, and are accepted with a probability of $\min \left[1, e^{-\delta \mathcal{H} / T}\right]$ with $\delta \mathcal{H}=-V_{0}\left[\cos \left(h_{i}^{\prime}\right)-\cos \left(h_{i}\right)\right]$. The parallel tempering algorithm considers then simultaneous copies of the system at different temperatures, allowing exchange of configurations between them. The exchange occurs after enough configurations have been generated at each temperature for a time greater than the energy autocorrelation time (see, e.g., Refs. [27,28] for details). This is particularly efficient for low-temperature configurations, which are most susceptible to being trapped in metastable regions. All the results reported in this paper have been obtained by means of this parallel tempering Monte Carlo algorithm, although we have checked that Langevin dynamics produces the same results (quantitatively within error bars).

Before proceeding with the discussion of the simulation results, let us recall the theoretical background. As we have already mentioned, the theorem proven in Ref. [12] implies that the system is in the rough phase at all nonzero temperatures. This can be interpreted in terms of general renormalization group arguments (for a renormalization group study of the 2D sine-Gordon model, see Refs. [22-24]; see also 


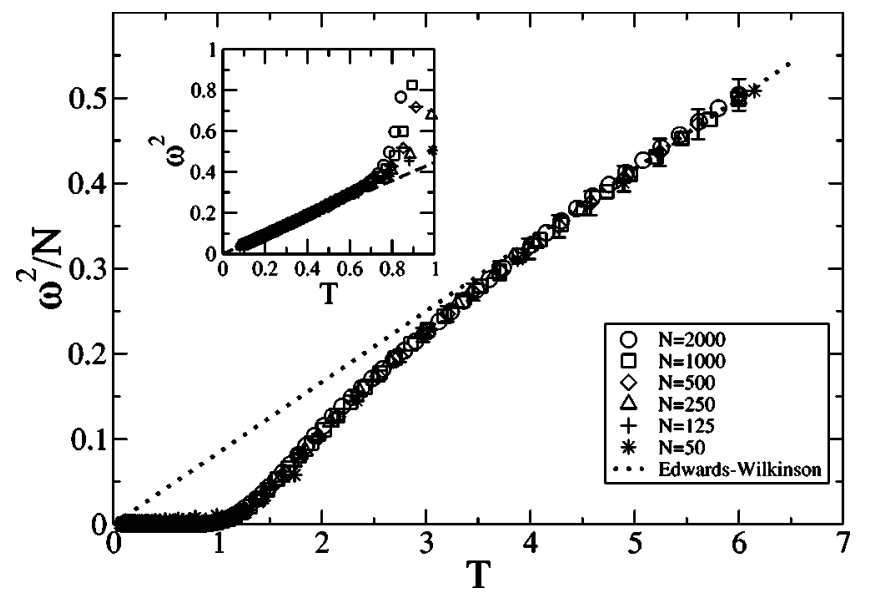

FIG. 1. Roughness scaled by the system size, $\omega^{2} / N$, vs temperature for the 1D sine-Gordon model. System sizes are as indicated in the figure. The dotted line corresponds to the theoretical value $\omega^{2} / N=T / 12$ obtained for the Edwards-Wilkinson model. Inset: zoom of the low temperature region showing the lack of scaling. The dashed line corresponds to the parabolical approximation discussed in the text, and is given by $\omega^{2}=T / \sqrt{V_{0}^{2}+4 V_{0}}$.

Refs. $[10,11]$ for a more general perspective): in the $1 \mathrm{D}$ model, fluctuations should be enough to effectively suppress the potential part of the Hamiltonian at any nonzero temperature, leaving as the only relevant term the discrete gradient (surface tension). In that case, the sine-Gordon model behaves effectively as the Edwards-Wilkinson model [29], whose associated Langevin equation (5) is simply the discrete linear diffusion equation with additive noise, and all the properties of interest can be calculated. This approach has been very successful in characterizing the $2 \mathrm{D}$ sine-Gordon model behavior [19], and particularly in locating the roughening transition temperature. In our $1 \mathrm{D}$ case, it is easy to show that in the Edwards-Wilkinson regime the roughness must scale linearly with the system size for nonzero temperature [30].

Figure 1 displays the simulation results for the roughness for several system sizes as a function of temperature. For the sake of definiteness we have chosen $J=V_{0}=1$ in Eq. (1); other choices yield the same qualitative results. Figure 1 shows that, as expected, the data tend asymptotically, for high values of the temperature, to the Edwards-Wilkinson result, $\omega^{2} / N=T / 12$ [30]. This linear scaling of the roughness with system size indicates clearly that the surface is rough at high temperatures. However, the main plot in the figure indicates a clear change of behavior around a temperature $T$ $\simeq 1$. In fact, as shown in the inset zoom, at low temperatures ( $T \lesssim 0.8$ ) the linear scaling dependence of the roughness with system size is lost and in that region the roughness becomes fairly independent of the system size, a behavior that according to the preceding discussion would correspond to a flat phase. To obtain a theoretical prediction for low temperatures, we have analyzed yet another linear model, in which the cosine term in the Hamiltonian is substituted by a parabolic potential, $V_{0}\left(1-\cos \left(h_{i}\right)\right) \rightarrow V_{0} h_{i}^{2} / 2$. Such a model is flat at all temperatures (basically because the parabolic potential confines the surface to lie around its minimum) and,

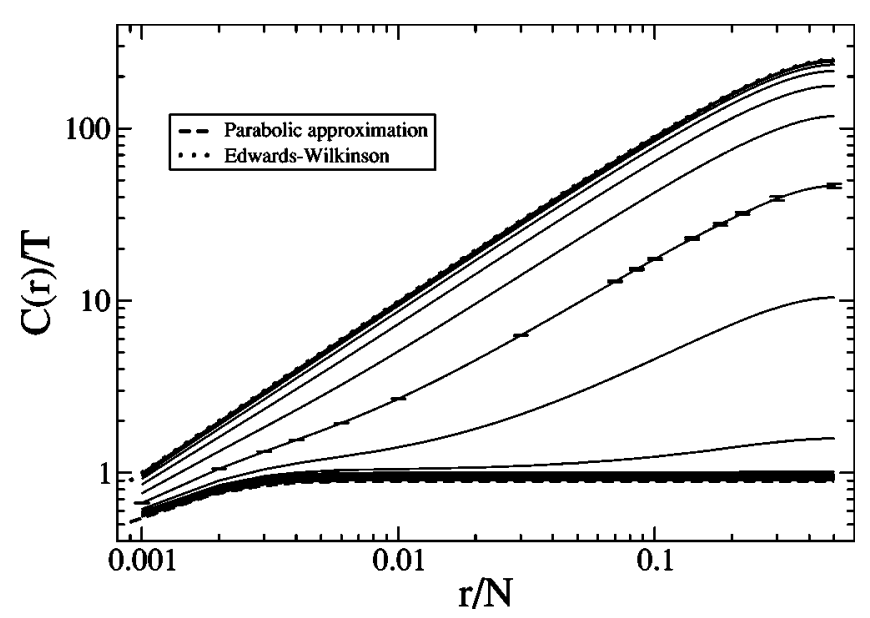

FIG. 2. Log-log plot of the height-difference correlation function scaled by temperature vs distance scaled by the system size. Bottom to top, temperatures are 0.0956, 0.2407, 0.7029, 0.8115, $0.9896,1.1689,1.4819,1.9016,2.5562,3.7044,6$. Also plotted are the predictions of the Edwards-Wilkinson model and of a parabolic approximation (see text). Error bars are typically as shown in one of the curves.

as can be observed in Fig. 1, agrees very well with the numerical simulations at low temperatures. We thus see that, in spite of the fact that we know that no phase transition can take place in this model, something very similar to a phase transition from a flat to a rough phase appears in the simulations for a temperature $T^{*} \simeq 0.8$.

The above indications are reinforced by looking at the correlation function, shown in Fig. 2, where we can see that for low temperatures there is a finite correlation length, whereas for high temperatures the correlation extends as far as half the system size (recall that we use periodic boundary conditions). It can also be appreciated again that for high temperatures the simulation results reproduce quite well the Edwards-Wilkinson prediction. In the opposite limit, much as it occurs with the roughness, the correlation behaves like the parabolic approximation. We have also studied other magnitudes, such as the specific heat, finding exactly the same result as Schneider and Stoll [14] that the specific heat exhibits a maximum at a value somewhat higher than $T^{*}$. We want to stress that all this is very reminiscent of the behavior in $2 \mathrm{D}$, where the maximum of the specific heat is interpreted as a Schottky anomaly (see Ref. [19] and references therein). Hence, from the available numerical evidence, we would be forced to conclude that there would be a roughening transition at a temperature $T^{*}$ in the $1 \mathrm{D}$ sineGordon model were it not for the theorem in Ref. [12].

\section{TRANSFER OPERATOR APPROACH}

In order to understand the numerical results, we will use the transfer operator approach $[1,9,14,15]$; specifically, to make the connection with the presentation in Ref. [12], we rewrite our Hamiltonian (1) rescaling the $h_{i}$ variables by a factor of $2 \pi$, i.e., 


$$
\mathcal{H}=\sum_{i=1}^{N}\left\{\frac{4 \pi^{2} J}{2}\left(h_{i-1}-h_{i}\right)^{2}+V_{0}\left[1-\cos \left(2 \pi h_{i}\right)\right]\right\} .
$$

In this notation, the preferred values for $h_{i}$ are the integer numbers, and the Hamiltonian is invariant under the transformation $h_{i} \mapsto h_{i}+1$. This means that we can choose for convenience $h_{1} \in\left(-\frac{1}{2}, \frac{1}{2}\right]$ without loss of generality. With this choice in mind, the corresponding partition function becomes

$$
\mathcal{Z}_{N}(\beta)=\int_{-1 / 2}^{1 / 2} d h_{1} \int_{-\infty}^{\infty} d h_{2} \cdots \int_{-\infty}^{\infty} d h_{N} e^{-\beta \mathcal{H}},
$$

$\beta$ being the inverse temperature in units of the Boltzmann constant. We now write $h_{i}=n_{i}+\phi_{i}$, with $n_{i} \in \mathbb{Z}$ and $-\frac{1}{2}$ $<\phi_{i} \leqslant \frac{1}{2}$, with $i=1, \ldots, N$. Let us define

$$
V(\beta, \phi, \theta) \equiv \sum_{n=-\infty}^{\infty} e^{-\beta 4 \pi^{2} J / 2(n+\phi)^{2}} e^{-\mathrm{i} n \theta},
$$

a $2 \pi$-periodic function of $\theta$, and the operator

$$
\begin{gathered}
\mathbf{T}_{\beta, \theta} f(\phi) \equiv \int_{-1 / 2}^{1 / 2} d \phi^{\prime} \mathcal{T}_{\beta, \theta}\left(\phi, \phi^{\prime}\right) f\left(\phi^{\prime}\right) \\
\mathcal{T}_{\beta, \theta}\left(\phi, \phi^{\prime}\right) \equiv V\left(\beta, \phi-\phi^{\prime}, \theta\right) \exp \left\{-\frac{\beta}{2} V_{0}[2-\cos (2 \pi \phi)\right. \\
\left.\left.-\cos \left(2 \pi \phi^{\prime}\right)\right]\right\}
\end{gathered}
$$

with these definitions, the partition function can be written as

$$
\mathcal{Z}_{N}(\beta)=\frac{1}{2 \pi} \int_{-\pi}^{\pi} d \theta \operatorname{Tr}\left(\mathbf{T}_{\beta, \theta}\right)^{N} .
$$

$\mathbf{T}_{\beta, \theta}$ is called the transfer operator for this model. Using the operator properties, it can be shown (see Ref. [12] for details) that in the thermodynamic limit $(N \rightarrow \infty)$,

$$
\begin{aligned}
\operatorname{Tr}\left(\mathbf{T}_{\beta, \theta}\right)^{N} & =\sum_{n \geqslant 1}\left[\lambda_{n}(\beta, \theta)\right]^{N} \\
& =m(\beta, \theta)\left[\lambda_{\max }(\beta, \theta)\right]^{N}[1+o(1)],
\end{aligned}
$$

where $\lambda_{n}$ are the operator eigenvalues, necessarily real and isolated, $\lambda_{\max }$ is the maximum eigenvalue, necessarily positive, and $m(\beta, \theta)$ is its multiplicity, necessarily finite. Finally, Laplace's method yields the free energy in the thermodynamic limit:

$$
-\beta f(\beta) \equiv \lim _{N \rightarrow \infty} \frac{1}{N} \ln \mathcal{Z}_{N}(\beta)=\max _{-\pi \leqslant \theta \leqslant \pi} \ln \left[\lambda_{\max }(\beta, \theta)\right] .
$$

Based on this expression, in Ref. [12] it was proven that the maximum of $\lambda_{\max }(\beta, \theta)$ occurs at $\theta=0$, and that $\lambda_{\max }(\beta, 0)$ is analytic for $\beta>0$, which leads to the conclusion that the free energy itself is analytic for all $\beta>0$ and, sub- sequently, that there are no phase transitions in the model. However, that is not all the information we can obtain from this approach, as we will now show.

In Ref. [14], the squared modulus of the eigenfunction of the largest eigenvalue is interpreted as the probability density for the $h_{i}$ variables. As the transfer operator in Ref. [14] is different from that we are using here, and for the sake of completeness, we now proceed to show that we can resort to the same interpretation here. Leaving out irrelevant constants, and keeping in mind that as we have just said the only contribution to the free energy comes from $\theta=0$, we can write

$$
\mathcal{Z}_{N}(\beta)=\operatorname{Tr}\left(\mathbf{T}_{\beta, 0}\right)^{N} .
$$

We can now compute averages of functions $g\left(\phi_{j}\right)$ in the following way [14]:

$$
\left\langle g\left(\phi_{j}\right)\right\rangle=\frac{\operatorname{Tr} \mathbf{T}_{\beta, 0}^{j} g\left(\phi_{j}\right) \mathbf{T}_{\beta, 0}^{N-j}}{\operatorname{Tr} \mathbf{T}_{\beta, 0}^{N}} .
$$

In the thermodynamic limit, it can be shown that the previous equation becomes

$$
\left\langle g\left(\phi_{j}\right)\right\rangle=\int_{-1 / 2}^{1 / 2} d \phi\left|\varphi_{0}(\phi)\right|^{2} g(\phi),
$$

where $\varphi_{0}(\phi)$ is the eigenfunction of the largest eigenvalue. We thus see that, indeed, $\left|\varphi_{0}(\phi)\right|^{2}$ can be understood as the probability density for the height to be in the interval $\left(-\frac{1}{2}, \frac{1}{2}\right]$ modulo 1 .

In order to apply this result, we must compute the eigenvalues and eigenfunctions of the transfer operator. This has to be done numerically: to this end, one has to discretize the operator and transform it into a matrix (see Refs. [9,14], see Ref. [31] for a detailed account). The advantage of the present formulation of the transfer formalism is that integrals are to be carried out on a finite interval, and therefore we do not need to introduce ad hoc any cutoff as in the case of integrals on an infinite interval, thus eliminating one possible source of error or inaccuracy. Specifically, in our numerical diagonalization procedure we have used $2001 \times 2001$ matrices; we have checked that increasing their size does not change the results significantly. As another check, we have computed the specific heat from the numerically computed eigenvalues, finding perfect agreement with the output of the simulations.

The inset of Fig. 3 shows the squared modulus of a typical eigenfunction at low temperature. The interpretation in terms of probability density indicates that probable values for the height lie close to the minimum of the potential, i.e., to $\phi$ $=0$, whereas values close to the maxima of the potential at $\phi= \pm 1 / 2$ are very unlikely to occur. We can associate the probability of taking a value of $\phi= \pm 1 / 2$ with the probability of formation of a kink or step, as once the height is at a maximum it can cross over to the neighboring potential well, thus giving rise to a kink. This interpretation suggests us to compare that estimate with the inverse of the system sizes studied in this work, which is a reasonable estimation of the 


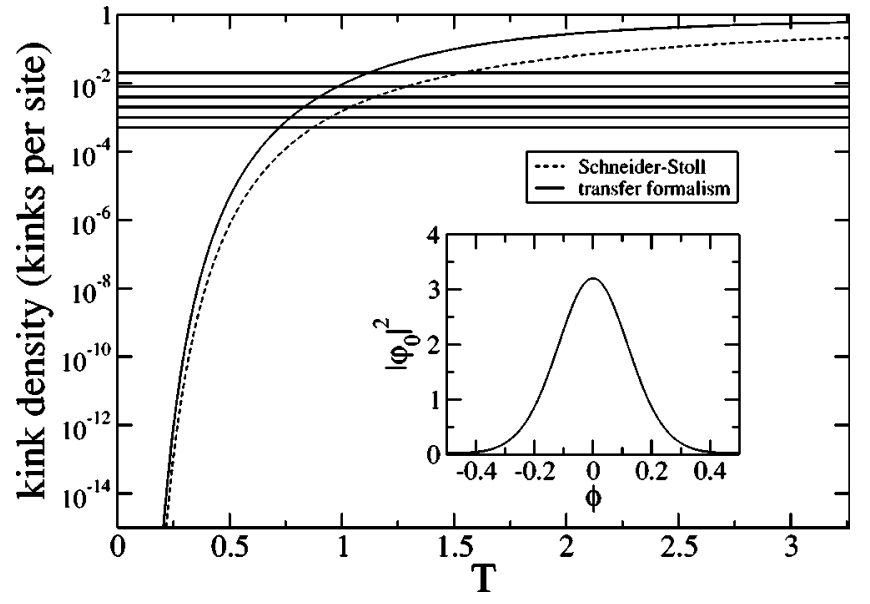

FIG. 3. Kink density as estimated from the probability that the value of the height goes over the potential maxima at $\phi= \pm 1 / 2$ (see text). Horizontal lines correspond to the inverses of the system sizes studied. For comparison, the approximate result obtained by Schneider and Stoll [14] is included as a dotted line. Inset: squared modulus of the eigenfunction of the largest eigenvalue at low temperature.

probability of observing a kink in our numerical system. Figure 3 compares both quantities, making clear that for every system size there is a temperature at which the probability of formation of one kink becomes smaller than the inverse of the system size. In fact, the probability of formation of one kink decays extremely rapidly below (orders of magnitude, note the logarithmic scale) the crossing temperature.

Following the discussion above, it is very natural then to associate that crossing temperature with $T^{*}$, the temperature at which we observe the apparent phase transition in our simulations. This is very well confirmed by Fig. 4, in which the roughness obtained from our numerical simulations is plotted along with the temperatures predicted by our crite-

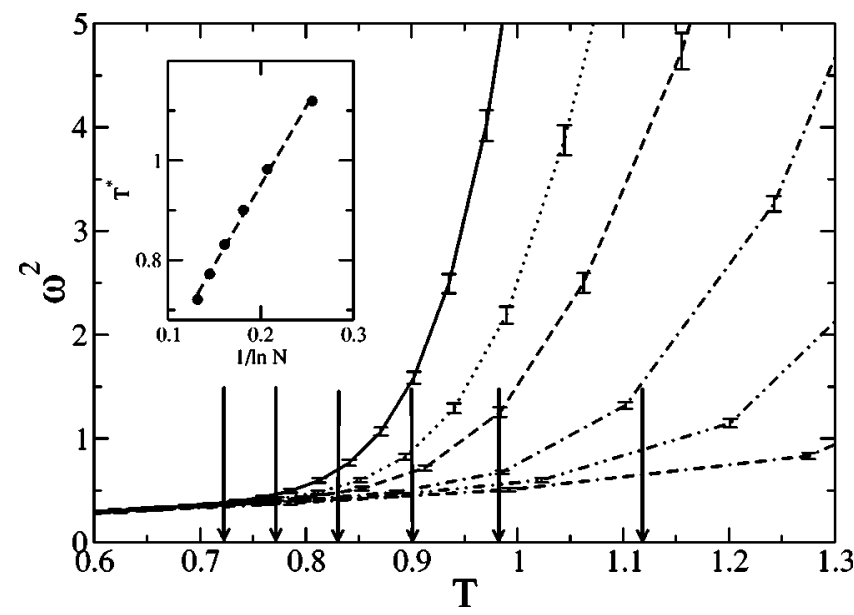

FIG. 4. Roughness vs temperature for different system sizes (right to left, $N=50,125,250,500,1000,2000$ ). The arrows mark the temperature predicted by our criterion based on the eigenfunction for each of those system sizes. Inset: estimates for $T^{*}$ vs $1 / \ln N$. Points are obtained from our criterion; the line is a linear regression fit. rion above. We note in passing that this result allows us to understand the reason why the crude parabolic approximation coincides so well with the numerical results at low temperatures: the hypothesis underlying the approximation is that kinks do not form and the whole surface lies close to a single potential minimum. Indeed, we have seen that the probability of kink formation at low temperatures is certainly negligible.

Summarizing, we have support for the criterion, as confirmed by the comparison of the transfer eigenfunction approach to the results of the numerical simulations, that the apparent transition temperature $T^{*}$ coincides with the one that yields a kink formation probability smaller than the inverse of the system size. This criterion allows us to make the following quantitative prediction: for a system of size $N$, there is an apparent flat-rough phase transition at a temperature $T^{*} \sim 1 / \ln N$, as shown in the inset of Fig. 4 . This can be easily understood if we realize that as kink formation is an activated phenomenon, the kink density follows an Arrhenius-type law (with corrections, see Ref. [14]). The dependence of $T^{*}$ on $N$ follows from our criterion by imposing the proportionality of the kink density and $1 / N$. We can now estimate the size of the system needed in order to observe the rough phase all the way down to any given temperature, $T^{*}$. Taking as an example $T^{*}=0.1$, which is certainly not small, we find that lattices of the order of $10^{30}$ sites are required to ensure a reasonable chance that kinks are formed during the simulation and the rough phase is observed for $T>T^{*}$; we would still find such an exceedingly large system in the flat phase for $T<T^{*}$. It is important to realize that the values we obtained for $T^{*}$ are likely to be as small as possible, because they come from a very efficient, parallel-tempering Monte Carlo algorithm that favors nucleation events through the exchange of configurations at different temperatures. Other procedures can perform worse leading to even larger values for $T^{*}$; this is the case, for instance, of Langevin dynamics simulations of the underdamped sine-Gordon equation with a damping term, as the nucleation rate decreases with increasing damping [32]. We have thus shown clearly that systems of any practically achievable size will always exhibit an apparent phase transition at a temperature far from $T=0$.

\section{CONCLUSIONS}

In this paper, we have studied analytically and numerically, by Langevin dynamics and mostly by parallel tempering Monte Carlo simulations, the 1D sine-Gordon model. We have found in the simulations that there exists a temperature at which an apparent roughening phase transition takes place. We have shown that it is possible to understand the contradiction of such phenomenon with the theorem that prohibits phase transitions in this model [12] through the analysis of the eigenfunctions of the corresponding transfer operator. The interpretation of these functions as probability densities makes clear that lattices of any finite size will always show an apparent phase transition, because the probability that kinks are formed becomes negligible below certain temperature. We have also seen that even in extremely large lattices 
the apparent transition occurs at temperatures far from zero and, in fact, $T^{*} \sim 1 / \ln N$.

The results summarized above are relevant in a much broader context, basically in two directions. First, our conclusion should be kept in mind when analyzing the outcome of numerical simulations of models about which there is little or none analytical information. Were it not for the fact that we know that such a phase transition is not possible, we would have concluded from our simulations that the 1D sineGordon model presents a roughening phase transition. In fact, simulations for the 2D sine-Gordon model yield results very similar to those presented here [19], although in that case we have a true phase transition according to several approximate calculations including renormalization group results $[16,22-24]$. It is important to realize that finite size analysis, which in principle could signal that the transition goes to $T=0$ with system size, becomes questionable if the values for the apparent transition at the sizes amenable within computational capabilities are still very far from $T$ $=0$. In addition, in our model, approximate analytical results in the low temperature limit support the existence of the nonexistent phase transition. Therefore, we conclude that one has to be extremely careful with claims of this kind.

The second direction that our work points to is related to the very nature of phase transitions. True thermodynamic phase transitions, understood as singularities of the free en- ergy or its derivatives, can only take place in the thermodynamic limit, and no such transitions occur in finite size systems. Hence, the apparent phase transition we see in our simulation can indeed be thought of as a true transition in the context of finite systems. What is more important, similar phenomenology is bound to arise in small, mesoscopic systems, certainly far from any thermodynamic limit one can think of. As systems of that scale become more and more relevant both for theoretical and for applied reasons, the question of the definition and nature of phase transitions gains importance. In this respect, this work hints that nonthermodynamic transitions may well be physically existent, or, alternatively, that computations and results in the thermodynamic limit do not represent well the fate of large but finite systems, even of very large, mesoscopic ones.

\section{ACKNOWLEDGMENTS}

S.A. and A.S. want to thank IMEDEA and Universitat de les Illes Balears for their hospitality during the progress of this work. S.A. and R.T. thank João M. V. P. Lopes for hospitality and discussions at Centro de Física do Porto. This work was supported by the Ministerio de Ciencia y Tecnología of Spain and FEDER through Grant Nos. BFM20000004 (J.A.C.), BFM2000-0006 (S.A. and A.S.), BFM20010341-C02-01, and BFM2000-1108 (R.T.).
[1] L. van Hove, Physica (Amsterdam) 16, 137 (1950) (reprinted in Ref. [3], p. 28).

[2] D. Ruelle, Statistical Mechanics: Rigorous Results (AddisonWesley, Reading, MA, 1989).

[3] Mathematical Physics in One Dimension, edited by E.H. Lieb and D.C. Mattis (Academic, New York, 1966).

[4] L.D. Landau and E.M. Lifshitz, Statistical Physics Part 1 (Pergamon, New York, 1980).

[5] J.F. Nagle, Am. J. Phys. 36, 1114 (1968).

[6] C. Kittel, Am. J. Phys. 37, 917 (1969).

[7] S.T. Chui and J.D. Weeks, Phys. Rev. B 23, 2438 (1981).

[8] T.W. Burkhardt, J. Phys. A 14, L63 (1981).

[9] T. Dauxois and M. Peyrard, Phys. Rev. E 51, 4027 (1995); T. Dauxois, N. Theodorakopoulos, and M. Peyrard, J. Stat. Phys. 107, 869 (2002).

[10] A.-L. Barabási and H.E. Stanley, Fractal Concepts in Surface Growth (Cambridge University, Cambridge, 1995).

[11] A. Pimpinelli and J. Villain, Physics of Crystal Growth (Cambridge University Press, Cambridge, 1998).

[12] J.A. Cuesta and A. Sánchez, J. Phys. A 35, 2373 (2002).

[13] M.E. Fisher and D.S. Fisher, Phys. Rev. B 25, 3192 (1982).

[14] T. Schneider and E. Stoll, Phys. Rev. B 22, 5317 (1980).

[15] T. Tsuzuki and K. Sasaki, Prog. Theor. Phys. Suppl. 94, 73 (1988)

[16] J.D. Weeks, in Ordering in Strongly Fluctuating Condensed Matter Systems, edited by T. Riste (Plenum, New York, 1980).

[17] F. Falo, A.R. Bishop, P.S. Lomdahl, and B. Horovitz, Phys. Rev. B 43, 8081 (1991).

[18] A. Sánchez, D. Cai, N. Grønbech-Jensen, A.R. Bishop, and Z.J. Wang, Phys. Rev. B 51, 14664 (1995); A. Sánchez, A.R.
Bishop, D. Cai, and N. Grønbech-Jensen, ibid. 52, 5433 (1995).

[19] A. Sánchez, A.R. Bishop, and E. Moro, Phys. Rev. E 62, 3219 (2000).

[20] R.A. Guyer and M.D. Miller, Phys. Rev. A 17, 1205 (1978).

[21] M. Büttiker and R. Landauer, Phys. Rev. Lett. 43, 1453 (1979); J. Phys. C 13, L325 (1980); Phys. Rev. A 23, 1397 (1981); Phys. Rev. B 24, 4079 (1981); in Nonlinear Phenomena at Phase Transitions and Instabilities, edited by T. Riste (Plenum, New York, 1982), p. 111.

[22] S.T. Chui and J.D. Weeks, Phys. Rev. B 14, 4978 (1976).

[23] H.J.F. Knops and L.W.J. den Ouden, Physica A 103, 597 (1980).

[24] P. Nozières, in Solids Far from Equilibrium, edited by C. Godréche (Cambridge University Press, Cambridge, 1991).

[25] M. San Miguel and R. Toral, in Instabilities and Nonequilibrium Structures VI, edited by E. Tirapegui, J. Martínez, and R. Tiemann (Kluwer Academic, Dordrecht, 2000).

[26] R. Toral, in Proceedings of the Third Granada Lectures in Computational Physics, edited by P.L. Garrido and J. Marro, Lecture Notes in Physics Vol. 448 (Springer-Verlag, Berlin, 1994).

[27] M.E.J. Newman and G.T. Barkema, Monte Carlo Methods in Statistical Physics (Oxford University, Oxford, 1999).

[28] Y. Iba, Int. J. Mod. Phys. C 12, 623 (2001).

[29] S.F. Edwards and D.R. Wilkinson, Proc. R. Soc. London, Ser. A 381, 17 (1982).

[30] B. Forrest and R. Toral, J. Stat. Phys. 70, 703 (1993).

[31] T. Dauxois, Ph.D. thesis, University of Dijon, 1993.

[32] S. Habib and G. Lythe, Phys. Rev. Lett. 84, 1070 (2000). 